OPEN ACCESS

Edited by:

Cheng-Chia Yu,

Chung Shan Medical

University, Taiwan

Reviewed by:

Philippe Gorphe,

Institut Gustave Roussy, France

Pei-ling Hsieh

China Medical University, Taiwan

*Correspondence:

Sumei Wang

wangsumei198708@163.com

Wanyin Wu

wwanyin@126.com

Specialty section:

This article was submitted to

Head and Neck Cancer,

a section of the journal

Frontiers in Oncology

Received: 01 April 2019

Accepted: 26 July 2019

Published: 13 August 2019

Citation:

Wang S, Claret F-X and Wu W (2019) MicroRNAs as Therapeutic Targets in

Nasopharyngeal Carcinoma.

Front. Oncol. 9:756

doi: 10.3389/fonc.2019.00756

\title{
MicroRNAs as Therapeutic Targets in Nasopharyngeal Carcinoma
}

\author{
Sumei Wang ${ }^{1,2,3 *}$, François-Xavier Claret $^{4,5}$ and Wanyin $W^{1,2 *}$ \\ ${ }^{1}$ Guangdong Provincial Key Laboratory of Clinical Research on Traditional Chinese Medicine Syndrome, Guangdong \\ Provincial Hospital of Chinese Medicine, Guangzhou, China, ${ }^{2}$ Department of Oncology, The Second Clinical College of \\ Guangzhou University of Chinese Medicine, Guangzhou, China, ${ }^{3}$ The Postdoctoral Research Station, Guangzhou University \\ of Chinese Medicine, Guangzhou, China, ${ }^{4}$ Department of Systems Biology, The University of Texas MD Anderson Cancer \\ Center, Houston, TX, United States, ${ }^{5}$ Experimental Therapeutic Academic Program and Cancer Biology Program, The \\ University of Texas Graduate School of Biomedical Sciences at Houston, Houston, TX, United States
}

Nasopharyngeal carcinoma (NPC) is a malignancy of epithelial origin that is prone to local invasion and early distant metastasis. Although concurrent chemotherapy and radiotherapy improves the 5-year survival outcomes, persistent or recurrent disease still occurs. Therefore, novel therapeutic targets are needed for NPC patients. MicroRNAs (miRNAs) play important roles in normal cell homeostasis, and dysregulations of miRNA expression have been implicated in human cancers. In NPC, studies have revealed that miRNAs are dysregulated and involved in tumorigenesis, metastasis, invasion, resistance to chemo- and radiotherapy, and other disease- and treatment-related processes. The advantage of miRNA-based treatment approaches is that miRNAs can concurrently target multiple effectors of pathways involved in tumor cell differentiation and proliferation. Thus, miRNA-based cancer treatments, alone or combined with standard chemotherapy and/or radiotherapy, hold promise to improve treatment response and cure rates. In this review, we will summarize the dysregulation of miRNAs in NPC initiation, progression, and treatment as well as NPC-related signaling pathways, and we will discuss the potential applications of miRNAs as biomarkers and therapeutic targets in NPC patients. We conclude that miRNAs might be potential promising therapeutic targets in nasopharyngeal carcinoma.

Keywords: microRNA, Nasopharyngeal Carcinoma (NPC), therapeutic target, biomarker, application

\section{INTRODUCTION}

\section{Nasopharyngeal Carcinoma (NPC)}

Nasopharyngeal carcinoma is a non-lymphomatous squamous cell carcinoma that arises from the epithelial lining of the nasopharynx. Local invasion and early distant metastasis are common in NPC. Etiologic factors for NPC include Epstein-Barr virus (EBV) infection, genetic predisposition, and environmental factors $(1,2)$. It is extremely difficult to detect early because of its deep location and lack of obvious clinical signs in its early stages. Concurrent chemotherapy and radiotherapy is a standard treatment for late-stage NPC (3). Nevertheless, despite the effectiveness of concurrent chemotherapy and radiotherapy in treating NPC, local or regional failure in the form of persistent or recurrent disease occurs in some patients. Therefore, novel biomarkers and therapeutic strategies to improve treatment outcomes are urgently required for NPC patients. 


\section{MicroRNAs (miRNAs)}

MiRNAs are a class of endogenous non-coding RNA molecules that are typically $22-25$ nucleotides long $(4,5)$. They are transcribed from intragenic or intergenic regions by RNA polymerase II into pri-miRNAs (at a length between 1 and $3 \mathrm{~kb}$ ) (6), and further processed by the RNase III ribonucleases Drosha and DiGeorge syndrome critical region gene 8, DGCR8, complex in the nucleus into a hairpin intermediate pre-miRNA (consisting in a stem-loop structure of about 70 nucleotides) (7). The premiRNA is then transported from the nucleus to the cytoplasm by exportin 5 (8). After strand separation, the mature doublestranded miRNA, also known as the guide strand, is incorporated into an RNA-induced silencing complex (RISC), whole the passenger strand (miRNA*) is typically degraded. The RISC is the effector complex of the miRNA pathway and comprises miRNA, Argonaute proteins (Argonaute 1 to Argonaute 4) and other proteins. The mature strand is important for target recognition and for the incorporation of specific target mRNAs into RISC $(8,9)$. Each miRNA can potentially target many genes (about 500 on average), and about $60 \%$ of mRNAs have at least 1 evolutionarily conserved sequence that is believed to be targeted by miRNAs $(10,11)$.

Usually, miRNAs target the 3 prime untranslated region ( 3 'UTR) of their target genes, most often causing mRNA deadenylation and degradation and subsequent translational repression $(5,12)$. However, other miRNA-mediated mechanisms of modulating mRNA expression have also been reported. Some miRNAs bind to the open reading frame or the $5^{\prime}$ UTR of their target genes; in some cases, miRNAs have been shown to activate gene expression rather than suppress it (13). For example, Jopling et al. reported that miR-122 can bind to 5'UTR, inhibiting translation of its target genes (14). In 2008, another miRNA, miR-10a, was reported to enhance translation by binding to ribosomal protein mRNA at the 5' UTR (which is known to regulate translation) downstream of the conserved 5 ' TOP motif (13). In 2016, we demonstrated that miR-24 could bind to both 3'UTR and 5'UTR of COPS5 (also named JAB1 and COP9 signalosome subunit 5), leading to COPS5 mRNA degradation and translational suppression (15). Besides, miRNAs can also

\footnotetext{
Abbreviations: A20/TNFAIP3, tumor necrosis factor alpha-induced protein 3; ABCB1, ATP binding cassette subfamily B member 1; ATM, ataxia telangiectasia mutated; BRD7, bromodomain containing 7; CAPN4/CAPNS1, calpain small subunit 1; circRNA, circular RNA; COPS5, COP9 signalosome subunit 5; CXCL12, C-X-C motif chemokine ligand 12; CXCR4, C-X-C motif chemokine receptor 4; DGCR8, Drosha and DiGeorge syndrome critical region gene 8; E2F3, E2F transcription factor 3; EBV, Epstein-Barr virus; EMT, epithelial-mesenchymal transition; EZH2, enhancer of zeste homolog 2; FGF5, fibroblast growth factor 5; IL-17, interleukin-17; ITGA3, integrin subunit alpha 3; KLF12, Kruppel-like factor 12; LASP1, LIM and SH3 protein 1; lncRNA, long non-coding RNA; MALAT1, metastasis-associated lung adenocarcinoma transcript-1; AP3K5/ASK1, apoptosis signal-regulating kinase1; miRNA, microRNA; MIF, macrophage migration inhibitory factor; mTOR, mechanistic target of rapamycin kinase; NF$\kappa \mathrm{B}$, nuclear factor $\kappa \mathrm{B}$; NPC, nasopharyngeal carcinoma; PDRG1, p53 and DNA damage regulated 1; PTEN, phosphatase and tensin homolog; RISC, RNA-induced silencing complex; RP, ribosomal protein; SP1, specificity protein 1; TIAM1, T cell lymphoma invasion and metastasis 1; TRIAP1, TP53-regulated inhibitor of apoptosis 1; TSGA10, testis-specific gene antigen 10; UTR, untranslated region; WNT2B, Wnt family member 2B; XIST, X inactivate-specific transcript.
}

regulate gene expression at the transcriptional level by binding directly to the DNA $(16,17)$. Moreover, proteins can also be targeted by miRNAs (18) (Figure 1).

\section{Exosomal miRNAs}

Exosomes are microvesicles that are $40-100 \mathrm{~nm}$ long. They originate in intracellular endosomal compartment and are secreted by cells into their microenvironment. Exosomes transport DNA fragments, proteins, mRNAs, and miRNAs from donor cells to recipient cells and are therefore crucial to intercellular communication. Exosomal miRNAs miR-21 and miR-29a are secreted by tumor cells and can bind to toll-like receptors on nearby immune cells, thus initiating an inflammatory response that promotes metastasis (19). Furthermore, miR-21 was observed at a higher level in exosomes from the serum of patients with esophageal squamous cell carcinoma than in serum from patients with benign diseases without systemic inflammation, and an association was found between exosomal miR-21 and the presence of metastasis with inflammation (20). In addition, exosomal miR-223 was reported to be elevated in breast cancer cells and promote breast cancer invasion (21). In NPC, exosomal miR-9 was found to inhibit angiogenesis through regulating PDK/AKT pathway (22). And exosomal miR-24-3p serves as a potential biomarker for NPC prognosis (23). Therefore, exosomal miRNAs are involved in the initiation and progression of cancers including NPC, and could be biomarkers for NPC patients.

\section{EBV-Encoded miRNAs}

EBV, a herpesvirus that infects the majority of the population worldwide asymptomatically (24), was the first human virus reported to encode miRNAs (25). More than 44 viral miRNAs are encoded from EBV. In NPC, EBV expresses EBNA1, LMP1, and LMP2A, EBERs, and BARTs (26). miR-BARTs, which are EBV-encoded miRNAs derived from BamH1-A rightward transcripts, are highly expressed in NPC and promote its development. A recent study showed that EBV-encoded miR-BARTS, including BART5-5p, BART7-3p, BART9-3p, and BART14-3p, downregulated the expression of a key DNA doublestrand break repair gene, ataxia telangiectasia mutated (ATM), by targeting several sites on its 3 '-UTR (27). Thus, those 4 EBVencoded miRNAs work cooperatively to suppress ATM activity in response to DNA damage, contributing to NPC tumorigenesis. Those findings indicate that EBV-encoded miRNAs can be used as a novel therapeutic strategy for NPC.

\section{THE MECHANISM OF MIRNA REGULATION IN CANCERS}

Genes encoding miRNAs are often located at or near fragile sites and in minimal regions of loss of heterozygosity, in minimal regions of amplification, and in common cancerrelated breakpoints (28). Upregulated expression of miRNAs can be caused by genomic alterations such as translocations o amplification, and loss of function can be caused by alterations such as deletions, insertions, or mutations (29). For example, the mir-17-92 cluster, which is made up of mir-17, mir-18a, mir-19b, 


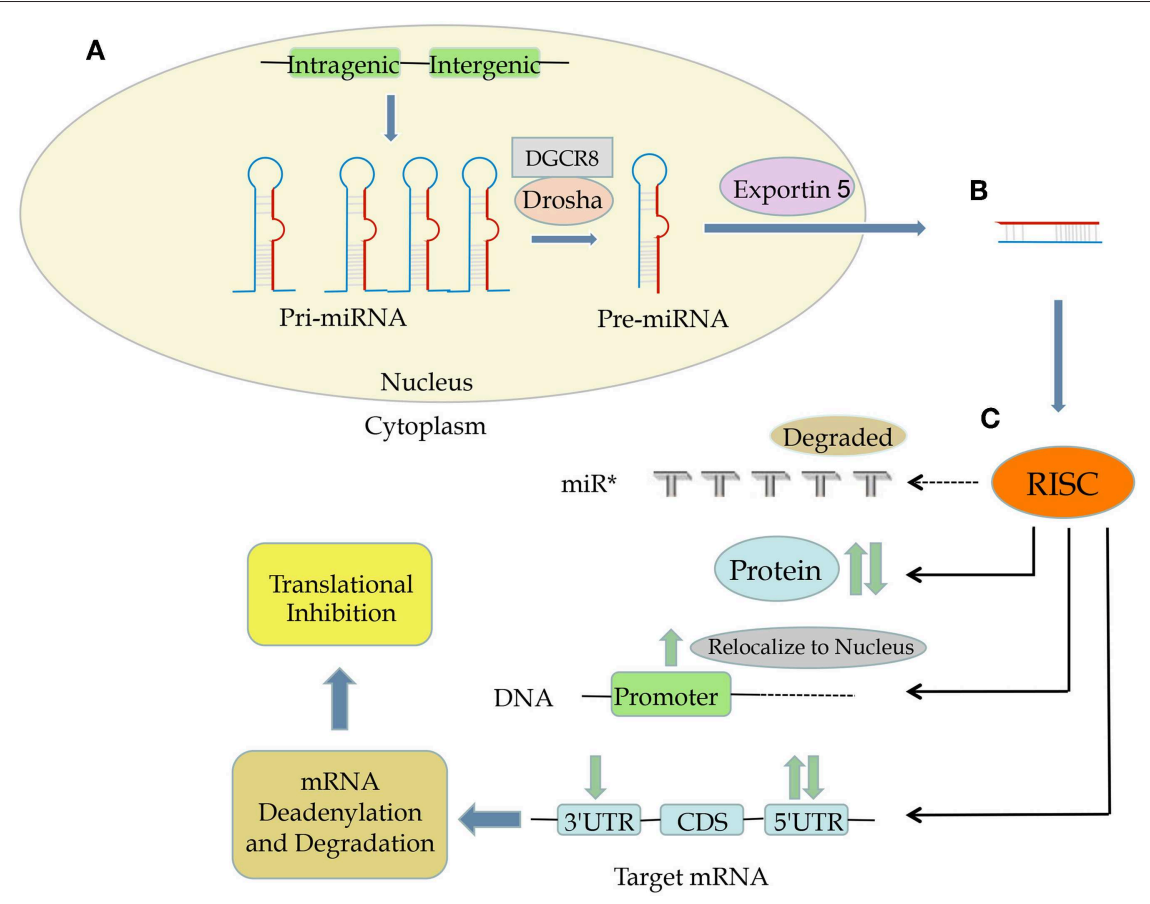

FIGURE 1 | The process and functions of miRNA. (A) Both intergenic and intragenic genes encode miRNAs. (B) Mature miRNAs are transcribed from pri-miRNA and then then pre-miRNA, translocated from nucleus to cytoplasm by exportin 5, and composed of RNA-induced silencing complex (RISC). (C) RISC can target mRNA at $3^{\prime}$ UTR, CDS, and 5' UTR as well as DNA promoter sequences and even proteins, leading to upregulation or downregulation of a specific protein.

mir-19b-1, mir-201, and mir-92-1, resides in an 800 base-pair region of the non-coding gene MIR17HG (also called C13orf25), a genomic region known to be amplified in lymphomas (30). The mir-17-92 cluster is often overexpressed in hematological cancers $(31,32)$. In contrast, the mir-15a-mir-16-a cluster, which resides in the chromosome 13q14 region (between exons 2 and 5 of the non-coding gene DLEU2), is often downregulated in patients with chronic lymphocytic leukemia due to genomic deletion of this region $(31,33)$.

In addition to structural genetic alterations, epigenetic modulations, including DNA promoter hypermethylation and histone hypoacetylation, have been described in solid tumors (34). For example, miR-127 is downregulated because of promoter hypermethylation in human bladder cancer (34). Usually, hypermethylation of tumor-suppressive miRNAs leads to miRNA silencing, and hypomethylation of onco-miRNAs leads to their activation and to tumorigenesis (35). In addition, long non-coding RNAs (lncRNAs) can target miRNAs, resulting in in tumorigenesis and chemo- and radioresistance. For example, lncRNA FTH1P3 promotes ATP binding cassette subfamily B member 1 (ABCB1) protein expression by targeting miR-206, acting as a miRNA "sponge," leading to the activation of paclitaxel resistance in breast cancer (36). Circular RNAs (circRNAs) also can act as miRNA sponges to regulate miRNA expression. For example, circNT5E was recently reported to directly bind to miR-422a and inhibit its activity, promoting glioblastoma tumorigenesis (37).

The aberrant miRNA expression in cancer can also be caused by downstream miRNA processing. Merritt et al. reported that miRNA expression could be globally suppressed by short hairpin
RNAs against Dicer and Drosha, 2 critical ribonucleases involved in miRNA processing (38). This miRNA suppression promotes cellular transformation and tumorigenesis.

The alteration of miRNA expression in cancers can also be caused by aberrant transcription factor activity, which leads to increased or decreased transcription from miRNA genes. The miR-34 miRNA family (comprising miR-34a, miR-34b, and miR$34 \mathrm{c}$ ) is directly induced by the tumor suppressor p53. In cells with high levels of p53, miR-34 expression is elevated; furthermore, chromatin immunoprecipitation assays revealed that p53 can bind to the promoter of miR-34 $(39,40)$. The MYC oncoprotein downregulates transcription of tumor suppressor miRNAs such as let-7 and miR-29 family members. MYC can bind to conserved sequences of the miRNA promoter that it suppresses, and the suppression of miRNAs by MYC has been found to facilitate lymphomagenesis (41) (Figure 2).

\section{MIRNA DYSREGULATION IN NPC INITIATION}

The function of miRNAs is largely influenced by the expression of their main targets. Some miRNAs promote tumorigenesis in some cell types and suppress it in others. The classification of a miRNA as an oncogene or a tumor suppressor, therefore, requires knowledge of the type of cell in which it acts. Typically, miRNAs do not cause a specific phenotype by aiming at a single target. Instead, miRNAs target multiple mRNAs concurrently and engage in complex interactions with the machinery that controls the transcriptome. In cancers, miRNAs often are 


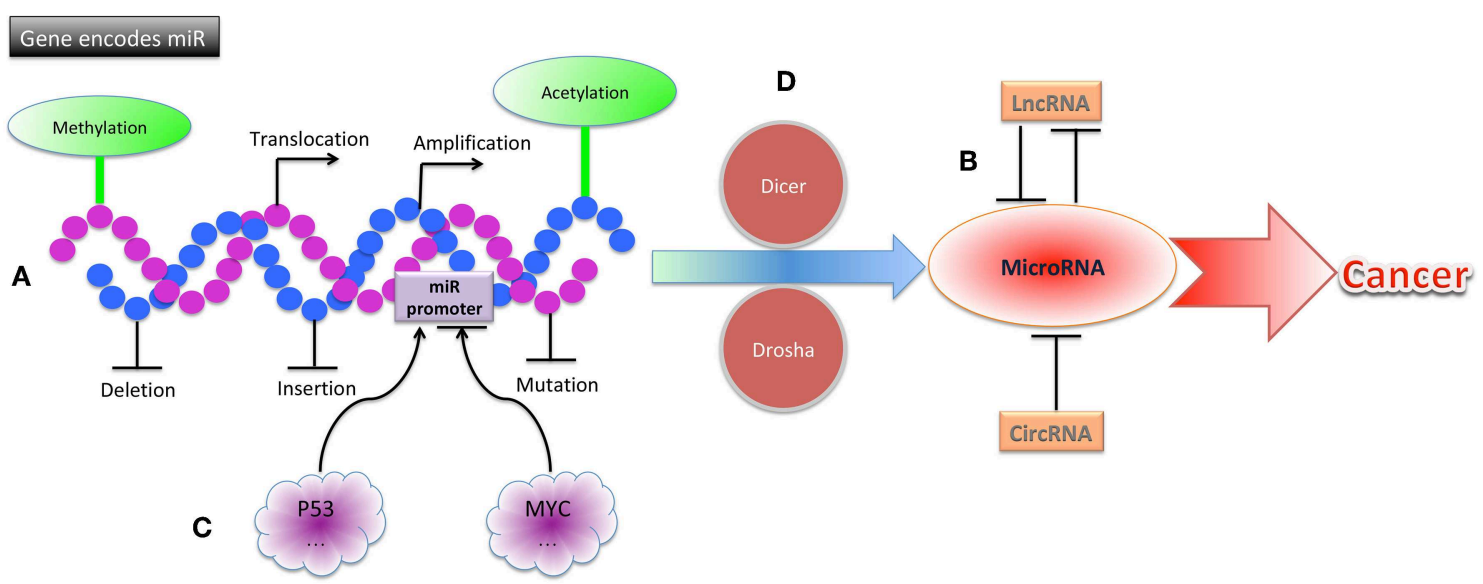

FIGURE 2 | The mechanisms of miRNA dysregulation in cancers. (A) Structural gene alterations, including translocation, amplification, deletion, insertion, and mutation contribute to miRNA dysregulation in cancers. (B) Epigenetic alterations, including methylation, acetylation, IncRNAs, and circRNAs contribute to miRNA dysregulation in cancers. (C) MiRNA promoters can be altered by p53, MYC, and other transcription factors, leading to cancer. (D) Alterations of proteins involved in miRNA processing, including Dicer, Drosha, and others, can result in cancer.

dysregulated and function collectively to mark differentiation states or individually as oncogenes or tumor suppressors. In NPC, miRNAs were reported to be expressed aberrantly and exert pivotal effects by altering the expression of their specific mRNA targets (42) (Table 1).

\section{Onco-miRNAs in NPC}

Tumor suppressor genes are usually inhibited by miRNAs directly, and such miRNAs are considered to be oncomiRNAs. For example, cell cycle inhibitor CDKN1A (also called P21) is believed to be directly targeted by miR-663 in NPC, leading to the promotion of NPC cell proliferation and tumorigenesis (43). An onco-miRNA, miR-125b, was found to be significantly upregulated in the NPC tissue compared with healthy nasopharyngeal mucosa, and this upregulation was correlated with poor survival outcomes; furthermore, high expression of miR-125b was identified as an independent predictor for shorter survival durations in NPC patients (44). The same study found that miR-125b promoted proliferation and inhibited apoptosis in NPC cells. A direct target of miR$125 \mathrm{~b}$, the tumor necrosis factor alpha-induced protein 3 gene TNFAIP3 (formerly called A20), functions as a tumor suppressor in NPC and mediates miR-125b-promoted NPC tumorigenesis by activating the nuclear factor $\kappa \mathrm{B}(\mathrm{NF}-\kappa \mathrm{B})$ signaling pathway. Together, these findings demonstrate that onco-miRNAs target tumor suppressor genes, leading to NPC initiation.

\section{Tumor-Suppressive miRNAs in NPC}

Oncogenes can be suppressed by miRNAs directly, and such miRNAs are considered to be tumor-suppressive miRNAs. For example, the C-X-C motif chemokine receptor 4 oncogene CXCR4 can be directly targeted by tumor-suppressive miR9, resulting in the inhibition of NPC pathogenesis. In NPC clinical specimens, miR-9 was observed to be downregulated (45). Interleukin-17 (IL-17), a proinflammatory cytokine, suppresses immune defense, and immune surveillance while promoting tumor growth. A study showed that IL-17 was targeted by miR-135a, resulting in the inhibition of NPC cell proliferation (46). In another study, overexpression of miR-320b was shown to suppress NPC cell proliferation and enhance mitochondrial fragmentation and apoptosis (47). In contrast, silencing miR$320 \mathrm{~b}$ enhanced NPC tumor growth and inhibited cell apoptosis. The TP53-regulated inhibitor of apoptosis 1 gene, TRIAP1, has been found to be directly targeted by miR-320b, which mediates TRIAP1's role in NPC cell proliferation inhibition and apoptosis induction. It has also been reported that miR-326/330$5 \mathrm{p}$ clusters can target cyclin D1 gene, CCND1, exerting their tumor-suppressive roles on NPC initiation (48). Some tumorsuppressive miRNAs target lncRNAs that function as oncogenes. For example, miR-25 expression was found to be upregulated in NPC cells, and its ectopic expression was shown to suppress NPC cell growth and motility by targeting metastasis-associated lung adenocarcinoma transcript-1 (MALAT1), a proto-oncogenic lncRNA (49). The tumor-suppressive miRNAs miR- 451 and miR-539-5p inhibit NPC initiation by targeting the macrophage migration inhibitory factor $(M I F)$ and Kruppel-like factor 12 (KLF12) genes, respectively $(50,51)$. Finally, lentivirus can be used as a delivery system to overexpress specific tumorsuppressive miRNAs in NPC, resulting in the inhibition of NPC initiation. For example, lenti-miR-26a was shown to inhibit the tumorigenicity of NPC cells in nude mice significantly, providing a useful strategy for treating NPC patients (52).

\section{MIRNA DYSREGULATION IN NPC PROGRESSION}

NPC is an aggressive disease that tends to spread locally and metastasize to regional lymph nodes and distant organs. Distant metastasis is the principal mode of treatment failure (53). 
TABLE 1 | miRNA dysregulation in NPC initiation, progression and therapies.

\begin{tabular}{|c|c|c|c|c|}
\hline miRNA & Target & Mechanism & Function & References \\
\hline miR-663 & CDKN1A & Promotes NPC cell proliferation & Onco-miRNA & $(43)$ \\
\hline miR-125b & TNFAIP3 & Inhibits NPC cell apoptosis & Onco-miRNA & $(44)$ \\
\hline miR-9 & CXCR4 & Suppresses NPC pathogenesis & Suppressive miRNA & $(45)$ \\
\hline miR-135a & IL-17 & Suppresses NPC cell proliferation & Suppressive miRNA & $(46)$ \\
\hline miR-320b & TRIAP1 & Enhances NPC cell apoptosis & Suppressive miRNA & $(47)$ \\
\hline miR-326/330-5p clusters & CCND1 & Suppresses NPC initiation & Suppressive miRNA & $(48)$ \\
\hline miR-25 & MALAT1 & Suppresses NPC cell growth & Suppressive miRNA & (49) \\
\hline Exosomal miR-23a & TSGA10 & Promotes NPC angiogenesis & Metastasis promoter & $(54)$ \\
\hline EBV-miR-BART1/7-3p & PTEN & Promotes EMT process & Metastasis promoter & $(55,56)$ \\
\hline miR-29c & TIAM1 & Suppresses NPC metastasis & Metastasis suppressor & $(57)$ \\
\hline miR-101 & ITGA3 & Inhibits NPC angiogenesis & Metastasis suppressor & $(58)$ \\
\hline miR-203a-3p & LASP1 & Inhibits NPC metastasis & Metastasis suppressor & (59) \\
\hline miR-630 & $E Z H 2$ & Inhibits NPC cell invasion & Metastasis suppressor & $(60)$ \\
\hline EBV-miR-BART6-3p & MIR3936HG & Suppresses NPC metastasis & Metastasis suppressor & $(61)$ \\
\hline miR-324-3p & WNT2B & Reduces NPC radioresistance & Radiosensitizer & (69) \\
\hline miR-519d & PDRG1 & Sensitizes NPC to IR & Radiosensitizer & $(70)$ \\
\hline miR-24 & COPS5/SP1 & Enhances NPC radiosensitivity & Radiosensitizer & $(15)$ \\
\hline miR-19b-3p & TNFAIP3 & Increases NPC radioresistance & Radioresistant agent & (63) \\
\hline miR-3188 & mTOR & Suppresses NPC resistance of 5-FU & Chemosensitizer & $(67)$ \\
\hline
\end{tabular}

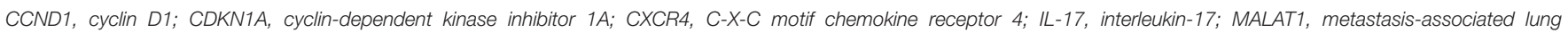

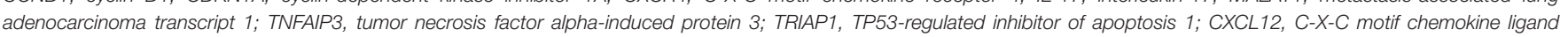

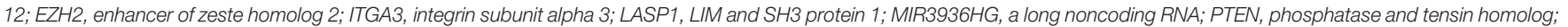

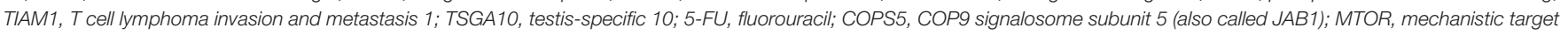
of rapamycin kinase; PDRG1, p53 and DNA damage regulated 1; SP1, specificity protein 1; WNT2B, Wnt family member $2 B$.

It is known that NPC metastasis is associated with miRNA dysregulation. The functions of miRNAs often are contradictory because they are determined by the cellular environment and the stage of the metastatic process. Therefore, identifying which miRNAs promote or suppress the metastatic process of NPC could lead to the development of new, efficient therapeutic agents to prevent or delay metastasis (Table 1).

\section{Metastasis Promoter miRNAs in NPC}

Mounting evidence implicates miRNAs in the modulation of angiogenesis, which is essential to the metastatic process. For instance, it has been reported that exosomal miR-23a overexpression promotes angiogenesis in NPC by directly targeting the testis-specific 10 gene, TSGA10 (54). Furthermore, miR-23a overexpression in pre-metastatic NPC tissue was identified as a prognostic biomarker for early metastasis. In addition, EBV-encoded miR-BART1 has been shown to induce NPC metastasis by regulating pathways that depend on the phosphatase and tensin homolog gene, PTEN (55). Another EBV-encoded miRNA, miR-BART7-3p, was shown to promote the epithelial-mesenchymal transition (EMT) and metastasis of NPC cells by suppressing PTEN and consequently activating the $\mathrm{PI} 3 \mathrm{~K} / \mathrm{AKT} / \mathrm{GSK}-3 \beta$ signaling pathway (56).

\section{Metastasis Suppressor miRNAs in NPC}

The tumor-suppressive miR-29c is also a metastasis suppressor that inhibits NPC cell migration, invasion, and metastasis by targeting the $\mathrm{T}$ cell lymphoma invasion and metastasis 1 gene,
TIAM1, directly (57). In NPC patient samples and cell lines, miR101 was found to be downregulated, and its ectopic expression significantly inhibited cell migration, invasion, and angiogenesis both in vitro and in vivo. The prometastatic gene integrin subunit alpha 3 (ITGA3) has been identified and validated as a target of miR-101 and shown to mediate the suppressive effects of miR101 on NPC metastasis. Interestingly, the systemic delivery of lentivirus-mediated miR-101 in NPC suppressed lung metastatic colony formation with no noticeable toxic effects (58). Also, miR203a-3p was found to be dysregulated and to act as a tumor suppressor in NPC. This miRNA suppresses NPC metastasis by targeting the LIM and SH3 protein 1 gene, LASP1 (59).

Metastasis suppressor miRNAs can also target or be targeted by $\operatorname{lncRNAs}$ in NPC. For example, lncRNA H19 has been found to be overexpressed in NPC tissue, and H19 knockdown significantly suppressed invasion NPC cells. H19 knockdown downregulated the expression of the enhancer of zeste homolog 2 gene, $E Z H 2$, which is upregulated in NPC and promotes invasion. $H 19$ does not bind directly to EZH2 but instead modulates its expression by suppressing the activity of miR-630, which inhibits EZH2 and interacts with $H 19$ in a sequencespecific manner. H19 also suppresses E-cadherin expression and promotes invasion in NPC cells through the miR-630/EZH2 pathway (60). And $\mathrm{He}$ et al. demonstrated that EBV-miRBART6-3p suppressed EBV-associated cancer cell migration and invasion by targeting lncRNA MIR3936HG (also known as LOC553103) and reversing the EMT process. And MIR3936HG knockdown by specific siRNAs was shown to phenocopy 
the effect of EBV-miR-BART6-3p, while elevated MIR3936HG expression enhanced tumor cell migration and invasion to promote EMT (61).

\section{MIRNA DYSREGULATION-RELATED SIGNALING PATHWAYS IN NPC}

Several signaling pathways are involved in miRNA dysregulationrelated processes in NPC. For example, miR-125b was shown to promote NPC tumorigenesis by activating the NF- $\kappa \mathrm{B}$ signaling pathway, which plays a critical role in NPC tumorigenesis and progression $(44,62)$. In addition, miR-19b-3p was found to be upregulated and to be an independent predictor for poor survival outcomes in NPC patients. MiR-19b-3b increased NPC cell radioresistance by targeting TNFAIP 3 and then activating the NF- $\kappa \mathrm{B}$ signaling pathway (63).

The PTEN/AKT pathway plays an important role in NPC processes related to miRNA dysregulation. One study found that miR-141 was markedly elevated in NPC tissues and negatively correlated with both patient survival and the expression of the bromodomain containing 7 gene, BRD7; BRD7 overexpression activated the PTEN/AKT pathway, but restoring miR-141 expression suppressed this activation and partially restored NPC cell proliferation and tumor growth. The BRD7/miR141/PTEN/AKT axis therefore is important to NPC progression and could provide new treatment targets and diagnostic markers (64). In addition, EBV-encoded miRNAs miR-BART1 and miR-BART7-3p promote NPC metastasis by modulating the PTEN/PI3K/AKT signaling pathway $(55,56)$. PI3K signaling is also involved in miRNA dysregulation-related processes in NPC. A study of NPC tumor specimens found that tumor-suppressing protein PDCD4 suppresses the pPI3K/pAKT/c-JUN signaling pathway, which in turn modulates miR-374a's binding to CCND1, resulting in dysregulation of NPC cell growth, metastasis, and chemoresistance (65). In this study, miR-374a expression was positively correlated with PDCD4 expression and negatively correlated with CCND1 expression. The PI3K/AKT/mTOR signaling pathway also significantly affects NPC tumorigenesis and development (66). For example, miR-3188 was shown to inhibit NPC cell cycle transition and proliferation, to sensitize cells to chemotherapy, and to extend survival in tumor-bearing mice, and to inactivate $\mathrm{p}-\mathrm{PI} 3 \mathrm{~K} / \mathrm{p}-\mathrm{AKT} / \mathrm{c}-\mathrm{JUN}$ signaling by targeting mTOR directly, further suppressing the cell cycle through the p-PI3K/p-AKT/p-mTOR pathway (67).

\section{MIRNA DYSREGULATION IN NPC THERAPIES}

Radiotherapy and chemotherapy are 2 main treatments for NPC. Mounting evidence shows that miRNAs are dysregulated during radio- or chemotherapy for NPC and may reduce or induce the sensitivity of NPC cells to radiotherapy or chemotherapy (Table 1).

\section{miRNA Dysregulation in Radiotherapy}

Radioresistance is the main reason for NPC treatment failure (68). Multiple studies have shown that miRNA expression in various cell types changed upon irradiation, as did the specific effects of various miRNAs on cellular radiosensitivity. It has been reported that miR-324-3p reduces NPC radioresistance by directly targeting the well-known oncogene Wnt family member 2B (WNT2B), inhibiting the gene's translation (69). Studies have also reported that miR-519d sensitizes NPC cells to radiation by directly targeting the $3^{\prime}$-UTR of PDRG1 (p53 and DNA damage regulated 1) mRNA (70) and thatiR-24 increases radiosensitivity in NPC by targeting both COPS5 and SP1 (specificity protein 1) (15). In contrast, Huang et al. demonstrated that miR-19b-3p upregulation decreases-and downregulation increases-NPC sensitivity to radiation. The researchers also found that miR-19b-3p directly targeted TNFAIP3, and the gene's upregulation reversed miR-19b-3p's suppressive effects on NPC cell radiosensitivity. Thus, miR-19b-3p was shown to enhance radioresistance in NPC cells by activating the TNFAIP3/ NF-кB pathway (63). Together, these studies indicate the potential use of miRNAs as radiosensitizing agents in NPC treatment.

\section{miRNAs Dysregulation in Chemotherapy}

The importance of miRNAs in chemotherapy response has been demonstrated in multiple human cancers, including cancer of the tongue (71). In NPC, miR-3188 has been found to inhibit cell growth and resistance to fluorouracil by directly targeting the mechanistic target of rapamycin kinase gene, $M T O R$, and regulating the cell cycle (67). Another study showed that the metastasis suppressor miR-29c can also increase NPC cells' sensitivity to both radiotherapy and cisplatin-based chemotherapy (72). The above evidence shows that miRNAs mainly function as chemosensitizers in NPC.

\section{mIRNAS AS BIOMARKERS AND NOVEL THERAPEUTIC APPROACHES IN NPC}

In the above sections, we showed that miRNAs are dysregulated during NPC initiation, progression, and therapy. In addition, several studies have reported that miRNA dysregulation is associated with the survival of NPC patients, and miRNAs may serve as independent biomarkers for NPC diagnosis, recurrence, and prognosis. Furthermore, a few molecularly targeted drugs have emerged as clinically active against advanced NPC in recent years (73), and the exploration of miRNAs as drugs or drug targets against other cancer types is already underway (29).

\section{miRNAs as Biomarkers in NPC}

Several miRNAs show potential as biomarkers in NPC. A recent meta-analysis indicated that increased miRNA expression led to a poor overall survival and increased the likelihood of death of NPC patients (74). The tumor and metastasis suppressor miR$29 \mathrm{c}$ has been shown to be downregulated in both the serum and tumor tissue of NPC patients, indicating its promise as a biomarker for NPC diagnosis, prognosis, and recurrence (75). Also, NPC patients were shown to have significantly higher serum levels of miR-663 compared with healthy individuals, and high levels were associated with worse 5-year overall and relapse-free survival outcomes in NPC patients (76). In addition, chemotherapy significantly lowered NPC patients' serum miR663 levels. These results suggest a critical role for miR-663 as 
a biomarker of NPC prognosis and response to chemotherapy. Recent studies showed that miR-31-5p was downregulated in present in NPC tissues and cell lines, acting as a tumor suppressive miRNA. And circulating miR-31-5p was identified to be a potential novel and non-invasive biomarker for the early diagnosis of NPC (77). The expression levels of tumoreducated platelet miR-34c-3p and miR-18a-5p are upregulated in NPC, which are promising novel liquid biopsy biomarkers for NPC diagnosis (78). In addition, miR-342-3p was significantly downregulated in NPC specimens and its low expression was significantly correlated with reduced overall survival of NPC patients, indicating miR-342-3p as a biomarker of NPC prognosis (79). All in all, the above results suggest miRNA can be a single biomarker for NPC diagnosis, prognosis, and response to therapy.

Additionally, miRNA signatures have been more and more identified to be novel biomarkers of NPC prognosis and prediction. For example, a two-miRNA signature of miR-548q and miR-483-5p were identified as potential biomarkers of NPC by comparing the plasma miRNA profiles of 31 NPC patients and 19 controls (80). And a three-miRNA signature including miR-548q, miR-630, and miR940 were increased in the plasma of NPC patients compared to those of controls. They are potential novel and useful biomarkers for NPC detection and diagnosis (81). The plasma level of miR-483-5p, miR-103, and miR-29a could be helpful to predict survival in patients with NPC (82). A four-miRNA signature of miR-22, miR-572, miR-638, and miR-1234 were identified to be prognostic biomarkers of NPC to the TNM staging system (83). Recently, a 8-miRNA signature including miR-188-5p, miR-1908, miR-3196, miR-3935, miR-4284, miR-4433-5p, miR-4665-3p, miR-513b, and 16-miRNA signature including miR-1224-3p, miR-1280, miR-155-5p, miR-1908, miR-1973, miR-296-5p,
miR-361-3p, miR-425-5p, miR-4284, miR-4436b-5p, miR-4439, miR-4665-3p, miR-4706, miR-4740-3p, miR-5091, miR-513b are promising biomarkers for NPC diagnosis (84). Taker together, miRNAs can be as both single biomarkers and signatures for NPC detection, diagnosis, and prognosis (Table 2).

\section{miRNAs as Therapeutic Approaches in NPC}

One of the most appealing properties of miRNAs as therapeutic agents is their ability to simultaneously target more than 1 gene, making miRNAs extremely efficient for regulating distinct cell processes relevant to normal and malignant cell homeostasis. In NPC, miRNAs for gene therapy have been delivered using lentiviral vectors. For example, a previous study found that tumor suppressor miR-31-5pinhibited EBVpositive NPC tumorigenesis, and minicircle-oriP-miR-31, a novel EBNA1-specific miRNA delivery system, was constructed and shown to inhibit NPC cell proliferation and migration in vitro and to suppress xenograft growth and lung metastasis in vivo. The researchers also found that the WD repeat domain 5 gene, WDR5, is a target of miR-31-5p. The study proved that targeted delivery of miR-31-5p using a non-viral minicircle vector could serve as a novel therapeutic approach for NPC, indicating a promising miRNA therapy for NPC patients (85). More studies are still ongoing to apply miRNA therapy to patients with NPC.

\section{CONCLUSIONS AND FUTURE DIRECTIONS}

The need for novel therapeutic targets and agents for treating NPC patients is urgent, owing to NPC's anatomical location and resistance to both radiotherapy and chemotherapy. Large

TABLE 2 | miRNAs as biomarkers in NPC

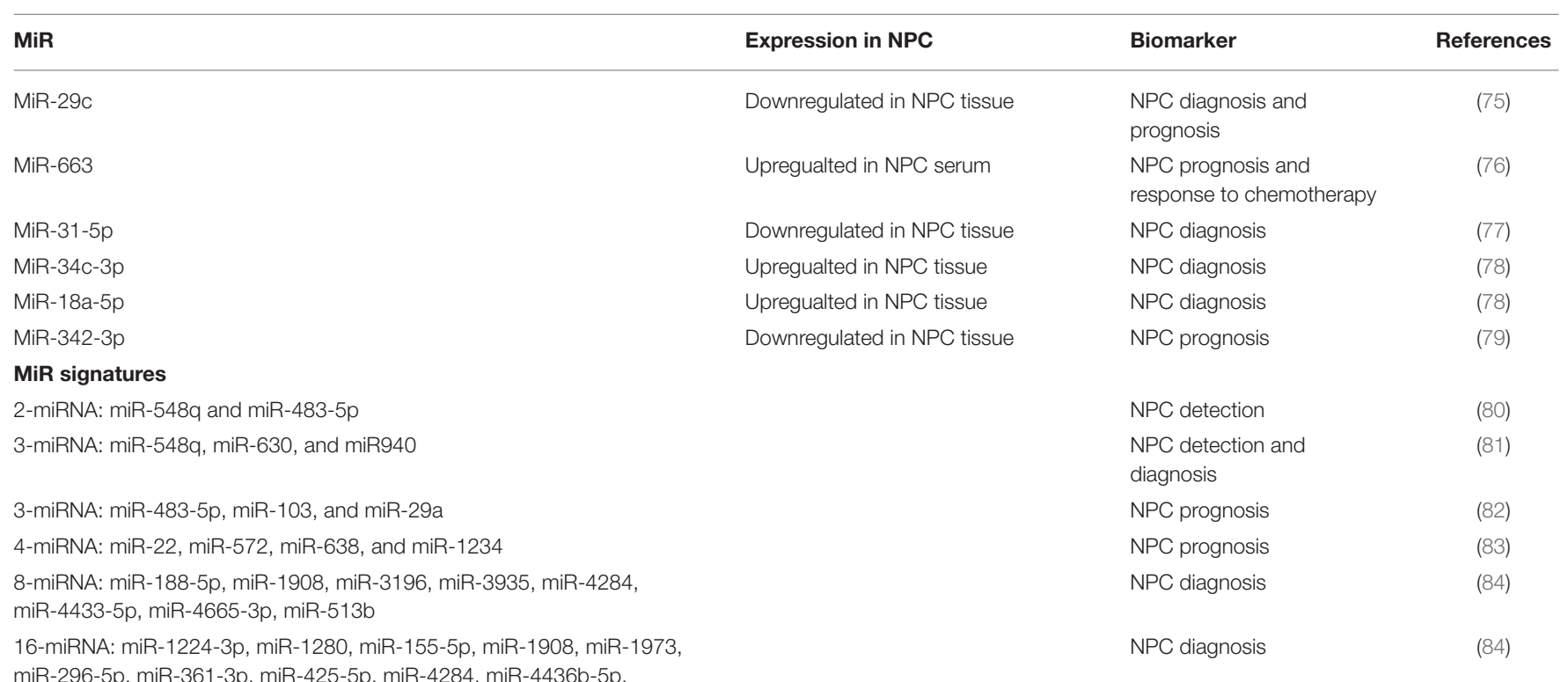

miR-4439, miR-4665-3p, miR-4706, miR-4740-3p, miR-5091, miR-513b 
numbers of miRNAs are dysregulated during the process of NPC initiation, progression, and therapy; therefore, miRNAs have been proposed as useful biomarkers to predict prognosis in and therapeutic approaches to cure patients with NPC. The advantages of using miRNAs as drugs antagonizing NPC is that 1 miRNA can target multiple targets and the same target can be targeted by many miRNAs, showing their comprehensive potential roles in the clinic. Based on the previous studies on miRNA dysregulations in NPC, how to use and take the advantages of miRNAs in clinic is to be solved. And due to the fact that miRNAs can regulate many mRNAs, the potential of toxic phenotypes and other off-target effects of miRNA treatment approaches is a major concern. As a result, more and more studies focusing on the toxic effects of targeting miRNAs are required before such therapies can be used safely in NPC patients.

\section{AUTHOR CONTRIBUTIONS}

SW was responsible for the writing and editing of the manuscript. F-XC was responsible for modifying the manuscript. WW provided some critical useful suggestions.

\section{REFERENCES}

1. Wei WI, Sham JS. Nasopharyngeal carcinoma. Lancet. (2005) 365:2041-54. doi: 10.1016/S0140-6736(05)66698-6

2. Le QT, Jones CD, Yau TK, Shirazi HA, Wong PH, Thomas EN, et al. A comparison study of different PCR assays in measuring circulating plasma epstein-barr virus DNA levels in patients with nasopharyngeal carcinoma. Clin Cancer Res. (2005) 11:5700-7. doi: 10.1158/1078-0432.CCR-05-0648

3. Chan AT, Gregoire V, Lefebvre JL, Licitra L, Felip E, Group E-E-EGW. Nasopharyngeal cancer: EHNS-ESMO-ESTRO Clinical Practice Guidelines for diagnosis, treatment and follow-up. Ann Oncol. (2010) 21(Suppl 5):v1879. doi: $10.1093 /$ annonc/mdq186

4. Lee RC, Feinbaum RL, Ambros V. The C. elegans heterochronic gene lin-4 encodes small RNAs with antisense complementarity to lin-14. Cell. (1993) 75:843-54. doi: 10.1016/0092-8674(93)90529-Y

5. Ambros V. The functions of animal microRNAs. Nature. (2004) 431:350-5. doi: 10.1038 /nature02871

6. Lee Y, Kim M, Han J, Yeom KH, Lee S, Baek SH, et al. MicroRNA genes are transcribed by RNA polymerase II. EMBO J. (2004) 23:4051-60. doi: 10.1038/sj.emboj.7600385

7. Lee Y, Ahn C, Han J, Choi H, Kim J, Yim J, et al. The nuclear RNase III Drosha initiates microRNA processing. Nature. (2003) 425:415-9. doi: 10.1038/nature01957

8. Bohnsack MT, Czaplinski K, Gorlich D. Exportin 5 is a RanGTP-dependent dsRNA-binding protein that mediates nuclear export of pre-miRNAs. RNA. (2004) 10:185-91. doi: 10.1261/rna.5167604

9. Chendrimada TP, Gregory RI, Kumaraswamy E, Norman J, Cooch N, Nishikura K, et al. TRBP recruits the Dicer complex to Ago2 for microRNA processing and gene silencing. Nature. (2005) 436:740-4. doi: 10.1038/nature03868

10. Betel D, Wilson M, Gabow A, Marks DS, Sander C. The microRNA.org resource: targets and expression. Nucleic Acids Res. (2008) 36:D149-53. doi: 10.1093/nar/gkm995

11. Friedman RC, Farh KK, Burge CB, Bartel DP. Most mammalian mRNAs are conserved targets of microRNAs. Genome Res. (2009) 19:92-105. doi: 10.1101/gr.082701.108

12. Lal A, Navarro F, Maher CA, Maliszewski LE, Yan N, O’Day E, et al. miR-24 Inhibits cell proliferation by targeting E2F2, MYC, and other cell-cycle genes

\section{FUNDING}

This work was supported by the Chinese medicine science and technology research project of Guangdong Provincial Hospital of Chinese Medicine (YN2016QJ03), the Guangdong Medical Science and Technology Research Foundation (A2018251), the doctoral research project of Guangdong Natural Science Foundation of China (2017A030310326), the China Postdoctoral Science Foundation Sixty-third Batches of Projects (2018M630941), the Guangzhou science and technology plan project (201804010149), the Major Research Projects in First-class Disciplines of Guangzhou University of Chinese Medicine (A1260619111001), and the Science and Technology Planning Project of Guangdong Province (2017B030314166).

\section{ACKNOWLEDGMENTS}

We thank Butt, Bryan F from the Department of Scientific Publications at The University of Texas MD Anderson Cancer Center for editorial support.

via binding to “seedless" 3'UTR microRNA recognition elements. Mol Cell. (2009) 35:610-25. doi: 10.1016/j.molcel.2009.08.020

13. Orom UA, Nielsen FC, Lund AH. MicroRNA-10a binds the 5'UTR of ribosomal protein mRNAs and enhances their translation. Mol Cell. (2008) 30:460-71. doi: 10.1016/j.molcel.2008.05.001

14. Jopling CL, Yi M, Lancaster AM, Lemon SM, Sarnow P. Modulation of hepatitis C virus RNA abundance by a liver-specific MicroRNA. Science. (2005) 309:1577-81. doi: 10.1126/science.11 13329

15. Wang S, Pan Y, Zhang R, Xu T, Wu W, Zhang R, et al. Hsa-miR-24$3 \mathrm{p}$ increases nasopharyngeal carcinoma radiosensitivity by targeting both the 3'UTR and 5'UTR of Jab1/CSN5. Oncogene. (2016) 35:6096-6108. doi: 10.1038 /onc.2016.147

16. Eiring AM, Harb JG, Neviani P, Garton C, Oaks JJ, Spizzo R, et al. miR328 functions as an RNA decoy to modulate hnRNP E2 regulation of mRNA translation in leukemic blasts. Cell. (2010) 140:652-65. doi: 10.1016/j.cell.2010.01.007

17. Place RF, Li LC, Pookot D, Noonan EJ, Dahiya R. MicroRNA-373 induces expression of genes with complementary promoter sequences. Proc Natl Acad Sci USA. (2018) 115:E3325. doi: 10.1073/pnas.1803 343115

18. Vasudevan S, Tong Y, Steitz JA. Switching from repression to activation: microRNAs can up-regulate translation. Science. (2007) 318:1931-4. doi: 10.1126/science. 1149460

19. Fabbri M, Paone A, Calore F, Galli R, Croce CM. A new role for microRNAs, as ligands of Toll-like receptors. RNA Biol. (2013) 10:169-74. doi: 10.4161/rna.23144

20. Tanaka Y, Kamohara H, Kinoshita K, Kurashige J, Ishimoto T, Iwatsuki M, et al. Clinical impact of serum exosomal microRNA-21 as a clinical biomarker in human esophageal squamous cell carcinoma. Cancer. (2013) 119:1159-67. doi: 10.1002/cncr.27895

21. Yang M, Chen J, Su F, Yu B, Su F, Lin L, et al. Microvesicles secreted by macrophages shuttle invasion-potentiating microRNAs into breast cancer cells. Mol Cancer. (2011) 10:117. doi: 10.1186/1476-4598-10-117

22. Lu J, Liu QH, Wang F, Tan JJ, Deng YQ, Peng XH, et al. Exosomal miR-9 inhibits angiogenesis by targeting MDK and regulating PDK/AKT pathway in nasopharyngeal carcinoma. J Exp Clin Cancer Res. (2018) 37:147. doi: 10.1186/s13046-018-0814-3 
23. Ye SB, Zhang H, Cai TT, Liu YN, Ni JJ, He J, et al. Exosomal miR-24$3 p$ impedes T-cell function by targeting FGF11 and serves as a potential prognostic biomarker for nasopharyngeal carcinoma. J Pathol. (2016) 240:329-40. doi: 10.1002/path.4781

24. Lo AK, To KF, Lo KW, Lung RW, Hui JW, Liao G, et al. Modulation of LMP1 protein expression by EBV-encoded microRNAs. Proc Natl Acad Sci USA. (2007) 104:16164-9. doi: 10.1073/pnas.0702896104

25. Pfeffer S, Zavolan M, Grasser FA, Chien M, Russo JJ, Ju J, et al. Identification of virus-encoded microRNAs. Science. (2004) 304:734-6. doi: 10.1126/science.1096781

26. Raab-Traub N. Epstein-Barr virus in the pathogenesis of NPC. Semin Cancer Biol. (2002) 12:431-41. doi: 10.1016/S1044579X0200086X

27. Lung RW, Hau PM, Yu KH, Yip KY, Tong JH, Chak WP, et al. EBV-encoded miRNAs target ATM-mediated response in nasopharyngeal carcinoma. $J$ Pathol. (2018) 244:394-407. doi: 10.1002/path.5018

28. Calin GA, Sevignani C, Dan Dumitru C, Hyslop T, Noch E, Yendamuri S, et al. Human microRNA genes are frequently located at fragile sites and genomic regions involved in cancers. Proc Natl Acad Sci USA. (2004) 101:2999-3004. doi: 10.1073/pnas.0307323101

29. Garzon R, Marcucci G, Croce CM. Targeting microRNAs in cancer: rationale, strategies and challenges. Nat Rev Drug Discov. (2010) 9:775-89. doi: $10.1038 / \mathrm{nrd} 3179$

30. Ota A, Tagawa H, Karnan S, Tsuzuki S, Karpas A, Kira S, et al. Identification and characterization of a novel gene, C13orf25, as a target for 13q31q32 amplification in malignant lymphoma. Cancer Res. (2004) 64:3087-95. doi: 10.1158/0008-5472.CAN-03-3773

31. Calin GA, Ferracin M, Cimmino A, Di Leva G, Shimizu M, Wojcik SE, et al. A MicroRNA signature associated with prognosis and progression in chronic lymphocytic leukemia. N Engl J Med. (2005) 353:1793-801. doi: 10.1056/NEJMoa050995

32. Garzon R, Volinia S, Liu CG, Fernandez-Cymering C, Palumbo T, Pichiorri F, et al. MicroRNA signatures associated with cytogenetics and prognosis in acute myeloid leukemia. Blood. (2008) 111:3183-9. doi: 10.1182/blood-2007-07-098749

33. Calin GA, Dumitru CD, Shimizu M, Bichi R, Zupo S, Noch E, et al. Frequent deletions and down-regulation of micro- RNA genes miR15 and miR16 at 13 q14 in chronic lymphocytic leukemia. Proc Natl Acad Sci USA. (2002) 99:15524-9. doi: 10.1073/pnas.242606799

34. Saito Y, Liang G, Egger G, Friedman JM, Chuang JC, Coetzee GA, et al. Specific activation of microRNA-127 with downregulation of the proto-oncogene BCL6 by chromatin-modifying drugs in human cancer cells. Cancer Cell. (2006) 9:435-43. doi: 10.1016/j.ccr.2006.04.020

35. Wang S, Wu W, Claret FX. Mutual regulation of microRNAs and DNA methylation in human cancers. Epigenetics. (2017) 12:187-97. doi: 10.1080/15592294.2016.1273308

36. Wang R, Zhang T, Yang Z, Jiang C, Seng J. Long non-coding RNA FTH1P3 activates paclitaxel resistance in breast cancer through miR-206/ABCB1. J Cell Mol Med. (2018) 22:4068-75. doi: 10.1111/jcmm.13679

37. Wang R, Zhang S, Chen X, Li N, Li J, Jia R, et al. CircNT5E acts as a sponge of microRNA-422a to promote glioblastoma tumorigenesis. Cancer Res. (2018) 78:4812-25. doi: 10.1158/0008-5472.CAN-18-0532

38. Merritt WM, Lin YG, Han LY, Kamat AA, Spannuth WA, Schmandt R, et al. Dicer, Drosha, and outcomes in patients with ovarian cancer. $N$ Engl J Med. (2008) 359:2641-50. doi: 10.1056/NEJMoa0803785

39. He L, He X, Lim LP, de Stanchina E, Xuan Z, Liang Y, et al. A microRNA component of the p53 tumour suppressor network. Nature. (2007) 447:11304. doi: 10.1038/nature05939

40. Chang TC, Wentzel EA, Kent OA, Ramachandran K, Mullendore M, Lee KH, et al. Transactivation of miR-34a by p53 broadly influences gene expression and promotes apoptosis. Mol Cell. (2007) 26:745-52. doi: 10.1016/j.molcel.2007.05.010

41. Chang TC, Yu D, Lee YS, Wentzel EA, Arking DE, West KM, et al. Widespread microRNA repression by Myc contributes to tumorigenesis. Nat Genet. (2008) 40:43-50. doi: 10.1038/ng.2007.30

42. Chen HC, Chen GH, Chen YH, Liao WL, Liu CY, Chang KP, et al. MicroRNA deregulation and pathway alterations in nasopharyngeal carcinoma. $\mathrm{Br} \mathrm{J}$ Cancer. (2009) 100:1002-11. doi: 10.1038/sj.bjc.6604948
43. Yi C, Wang Q, Wang L, Huang Y, Li L, Liu L, et al. MiR-663, a microRNA targeting p21(WAF1/CIP1), promotes the proliferation and tumorigenesis of nasopharyngeal carcinoma. Oncogene. (2012) 31:4421-33. doi: 10.1038/onc.2011.629

44. Zheng Z, Qu JQ, Yi HM, Ye X, Huang W, Xiao T, et al. MiR-125b regulates proliferation and apoptosis of nasopharyngeal carcinoma by targeting A20/NF-kappaB signaling pathway. Cell Death Dis. (2017) 8:e2855. doi: $10.1038 /$ cddis.2017.211

45. Lu J, Luo H, Liu X, Peng Y, Zhang B, Wang L, et al. miR-9 targets CXCR4 and functions as a potential tumor suppressor in nasopharyngeal carcinoma. Carcinogenesis. (2014) 35:554-63. doi: 10.1093/carcin/bgt354

46. Wang LX, Kang ZP, Yang ZC, Ma RX, Tan Y, Peng XB, et al. MicroRNA$135 \mathrm{a}$ inhibits nasopharyngeal carcinoma cell proliferation through targeting interleukin-17. Cell Physiol Biochem. (2018) 46:2232-8. doi: 10.1159/000489591

47. Li Y, Tang X, He Q, Yang X, Ren X, Wen X, et al. Overexpression of mitochondria mediator gene TRIAP1 by miR-320b loss is associated with progression in nasopharyngeal carcinoma. PLoS Genet. (2016) 12:e1006183. doi: 10.1371/journal.pgen.1006183

48. Song P, Yin SC. Long non-coding RNA EWSAT1 promotes human nasopharyngeal carcinoma cell growth in vitro by targeting miR-326/-330-5p. Aging. (2016) 8:2948-60. doi: 10.18632/aging.101103

49. Hua WF, Zhong Q, Xia TL, Chen Q, Zhang MY, Zhou AJ, et al. RBM24 suppresses cancer progression by upregulating miR-25 to target MALAT1 in nasopharyngeal carcinoma. Cell Death Dis. (2016) 7:e2352. doi: $10.1038 /$ cddis.2016.252

50. Liu N, Jiang $\mathrm{N}$, Guo R, Jiang $\mathrm{W}$, He QM, Xu YF, et al. MiR-451 inhibits cell growth and invasion by targeting MIF and is associated with survival in nasopharyngeal carcinoma. Mol Cancer. (2013) 12:123. doi: 10.1186/1476-4598-12-123

51. Sun KY, Peng T, Chen Z, Song P, Zhou XH. Long non-coding RNA LOC100129148 functions as an oncogene in human nasopharyngeal carcinoma by targeting miR-539-5p. Aging. (2017) 9:999-1011. doi: 10.18632/aging.101205

52. Lu JA, He ML, Wang L, Chen Y, Liu XO, Dong Q, et al. MiR26 a inhibits cell growth and tumorigenesis of nasopharyngeal carcinoma through repression of EZH2. Cancer Res. (2011) 71:225-33. doi: 10.1158/0008-5472.CAN-10-1850

53. Li ZL, Ye SB, OuYang LY, Zhang H, Chen YS, He J, et al. COX-2 promotes metastasis in nasopharyngeal carcinoma by mediating interactions between cancer cells and myeloid-derived suppressor cells. Oncoimmunology. (2015) 4:e1044712. doi: 10.1080/2162402X.2015.1044712

54. Bao L, You B, Shi S, Shan Y, Zhang Q, Yue H, et al. Metastasis-associated miR23a from nasopharyngeal carcinoma-derived exosomes mediates angiogenesis by repressing a novel target gene TSGA10. Oncogene. (2018) 37:2873-89. doi: 10.1038/s41388-018-0183-6

55. Cai L, Ye Y, Jiang Q, Chen Y, Lyu X, Li J, et al. Epstein-Barr virusencoded microRNA BART1 induces tumour metastasis by regulating PTENdependent pathways in nasopharyngeal carcinoma. Nat Commun. (2015) 6:7353. doi: 10.1038/ncomms8353

56. Cai LM, Lyu XM, Luo WR, Cui XF, Ye YF, Yuan CC, et al. EBV-miR-BART7$3 \mathrm{p}$ promotes the EMT and metastasis of nasopharyngeal carcinoma cells by suppressing the tumor suppressor PTEN. Oncogene. (2015) 34:2156-66. doi: 10.1038/onc.2014.341

57. Liu N, Tang LL, Sun Y, Cui RX, Wang HY, Huang BJ, et al. MiR-29c suppresses invasion and metastasis by targeting TIAM1 in nasopharyngeal carcinoma. Cancer Lett. (2013) 329:181-8. doi: 10.1016/j.canlet.2012.10.032

58. Tang XR, Wen X, He QM, Li YQ, Ren XY, Yang XJ, et al. MicroRNA-101 inhibits invasion and angiogenesis through targeting ITGA3 and its systemic delivery inhibits lung metastasis in nasopharyngeal carcinoma. Cell Death Dis. (2017) 8:e2566. doi: 10.1038/cddis.2016.486

59. Jiang $\mathrm{N}$, Jiang $\mathrm{X}$, Chen Z, Song X, Wu L, Zong D, et al. MiR-203a$3 \mathrm{p}$ suppresses cell proliferation and metastasis through inhibiting LASP1 in nasopharyngeal carcinoma. J Exp Clin Cancer Res. (2017) 36:138. doi: 10.1186/s13046-017-0604-3

60. Li X, Lin Y, Yang X, Wu X, He X. Long noncoding RNA H19 regulates $\mathrm{EZH} 2$ expression by interacting with miR-630 and promotes cell invasion in 
nasopharyngeal carcinoma. Biochem Biophys Res Commun. (2016) 473:913-9. doi: 10.1016/j.bbrc.2016.03.150

61. He B, Li W, Wu Y, Wei F, Gong Z, Bo H, et al. Epstein-Barr virusencoded miR-BART6-3p inhibits cancer cell metastasis and invasion by targeting long non-coding RNA LOC553103. Cell Death Dis. (2016) 7:e2353. doi: $10.1038 /$ cddis.2016.253

62. Zhu DD, Zhang J, Deng W, Yip YL, Lung HL, Tsang CM, et al. Significance of NF-kappaB activation in immortalization of nasopharyngeal epithelial cells. Int J Cancer. (2016) 138:1175-85. doi: 10.1002/ijc.29850

63. Huang T, Yin L, Wu J, Gu JJ, Wu JZ, Chen D, et al. MicroRNA-19b$3 \mathrm{p}$ regulates nasopharyngeal carcinoma radiosensitivity by targeting TNFAIP3/NF-kappaB axis. J Exp Clin Cancer Res. (2016) 35:188. doi: 10.1186/s13046-016-0465-1

64. Liu Y, Zhao R, Wang H, Luo Y, Wang X, Niu W, et al. miR-141 is involved in BRD7-mediated cell proliferation and tumor formation through suppression of the PTEN/AKT pathway in nasopharyngeal carcinoma. Cell Death Dis. (2016) 7:e2156. doi: 10.1038/cddis.2016.64

65. Zhen Y, Fang W, Zhao M, Luo R, Liu Y, Fu Q, et al. miR-374aCCND1-pPI3K/AKT-c-JUN feedback loop modulated by PDCD4 suppresses cell growth, metastasis, and sensitizes nasopharyngeal carcinoma to cisplatin. Oncogene. (2017) 36:275-85. doi: $10.1038 /$ onc.20 16.201

66. Wang F, Lu J, Peng X, Wang J, Liu X, Chen X, et al. Integrated analysis of microRNA regulatory network in nasopharyngeal carcinoma with deep sequencing. J Exp Clin Cancer Res. (2016) 35:17. doi: 10.1186/s13046-016-0292-4

67. Zhao M, Luo R, Liu Y, Gao L, Fu Z, Fu Q, et al. miR-3188 regulates nasopharyngeal carcinoma proliferation and chemosensitivity through a FOXO1-modulated positive feedback loop with mTOR-pPI3K/AKT-c-JUN. Nat Commun. (2016) 7:11309. doi: 10.1038/ncomms 11309

68. Liu SC, Tsang NM, Chiang WC, Chang KP, Hsueh C, Liang Y, et al. Leukemia inhibitory factor promotes nasopharyngeal carcinoma progression and radioresistance. J Clin Invest. (2013) 123:5269-83. doi: 10.1172/JCI 63428

69. Li G, Liu Y, Su Z, Ren S, Zhu G, Tian Y, et al. MicroRNA-324-3p regulates nasopharyngeal carcinoma radioresistance by directly targeting WNT2B. Eur J Cancer. (2013) 49:2596-607. doi: 10.1016/j.ejca.2013. 03.001

70. $\mathrm{Xu} \mathrm{T}$, Xiao D. Oleuropein enhances radiation sensitivity of nasopharyngeal carcinoma by downregulating PDRG1 through HIF1alpha-repressed microRNA-519d. J Exp Clin Cancer Res. (2017) 36:3. doi: 10.1186/s13046-016-0480-2

71. Sun L, Yao Y, Liu B, Lin Z, Lin L, Yang M, et al. MiR-200b and miR$15 \mathrm{~b}$ regulate chemotherapy-induced epithelial-mesenchymal transition in human tongue cancer cells by targeting BMI1. Oncogene. (2012) 31:432-45. doi: 10.1038/onc.2011.263

72. Zhang JX, Qian D, Wang FW, Liao DZ, Wei JH, Tong ZT, et al. MicroRNA$29 \mathrm{c}$ enhances the sensitivities of human nasopharyngeal carcinoma to cisplatin-based chemotherapy and radiotherapy. Cancer Lett. (2013) 329:91-8. doi: 10.1016/j.canlet.2012.10.033

73. Lim WT, Ng QS, Ivy P, Leong SS, Singh O, Chowbay B, et al. A Phase II study of pazopanib in Asian patients with recurrent/metastatic nasopharyngeal carcinoma. Clin Cancer Res. (2011) 17:5481-9. doi: 10.1158/1078-0432.CCR-10-3409
74. Sabarimurugan S, Kumarasamy C, Baxi S, Devi A, Jayaraj R. Systematic review and meta-analysis of prognostic microRNA biomarkers for survival outcome in nasopharyngeal carcinoma. PLoS ONE. (2019) 14:e0209760. doi: 10.1371/journal.pone.0209760

75. Niu M, Gao D, Wen Q, Wei P, Pan S, Shuai C, et al. MiR-29c regulates the expression of miR-34c and miR-449a by targeting DNA methyltransferase $3 \mathrm{a}$ and $3 \mathrm{~b}$ in nasopharyngeal carcinoma. BMC Cancer. (2016) 16:218. doi: 10.1186/s12885-016-2253-x

76. Liang S, Zhang N, Deng Y, Chen L, Zhang Y, Zheng Z, et al. Increased serum level of microRNA-663 is correlated with poor prognosis of patients with nasopharyngeal carcinoma. Dis Markers. (2016) 2016:7648215. doi: 10.1155/2016/7648215

77. Yi SJ, Liu P, Chen BL, Ou-Yang L, Xiong WM, Su JP. Circulating miR-31$5 \mathrm{p}$ may be a potential diagnostic biomarker in nasopharyngeal carcinoma. Neoplasma. (2019) 10:181109N847.doi: 10.4149/neo_2018_181109N847

78. Wang H, Wei X, Wu B, Su J, Tan W, Yang K. Tumor-educated platelet miR-34c-3p and miR-18a-5p as potential liquid biopsy biomarkers for nasopharyngeal carcinoma diagnosis. Cancer Manag Res. (2019) 11:3351-60. doi: 10.2147/CMAR.S195654

79. Cui Z, Zhao Y. microRNA-342-3p targets FOXQ1 to suppress the aggressive phenotype of nasopharyngeal carcinoma cells. BMC Cancer. (2019) 19:104. doi: 10.1186/s12885-018-5225-5

80. Zheng XH, Cui C, Ruan HL, Xue WQ, Zhang SD, Hu YZ, et al. Plasma microRNA profiling in nasopharyngeal carcinoma patients reveals miR-548q and miR-483-5p as potential biomarkers. Chin J Cancer. (2014) 33:330-8. doi: $10.5732 /$ cjc. 013.10246

81. Zhuo X, Zhou W, Li D, Chang A, Wang Y, Wu Y, et al. Plasma microRNA expression signature involving miR-548q, miR-630 and miR-940 as biomarkers for nasopharyngeal carcinoma detection. Cancer Biomarkers. (2018) 23:579-87. doi: 10.3233/CBM-181852

82. Wang HY, Yan LX, Shao Q, Fu S, Zhang ZC, Ye W, et al. Profiling plasma microRNA in nasopharyngeal carcinoma with deep sequencing. Clin Chem. (2014) 60:773-82. doi: 10.1373/clinchem.2013.214213

83. Liu N, Cui RX, Sun Y, Guo R, Mao YP, Tang LL, et al. A four-miRNA signature identified from genome-wide serum miRNA profiling predicts survival in patients with nasopharyngeal carcinoma. Int J Cancer. (2014) 134:1359-68. doi: 10.1002/ijc. 28468

84. Wen W, Mai SJ, Lin HX, Zhang MY, Huang JL, Hua X, et al. Identification of two microRNA signatures in whole blood as novel biomarkers for diagnosis of nasopharyngeal carcinoma. J Transl Med. (2019) 17:186. doi: 10.1186/s12967-019-1923-2

85. Wu J, Tan X, Lin J, Yuan L, Chen J, Qiu L, et al. Minicircle-oriP-miR31 as a novel EBNA1-specific miRNA therapy approach for nasopharyngeal carcinoma. Hum Gene Ther. (2017) 28:415-27. doi: 10.1089/hum.2016.136

Conflict of Interest Statement: The authors declare that the research was conducted in the absence of any commercial or financial relationships that could be construed as a potential conflict of interest.

Copyright (c) 2019 Wang, Claret and Wu. This is an open-access article distributed under the terms of the Creative Commons Attribution License (CC BY). The use, distribution or reproduction in other forums is permitted, provided the original author(s) and the copyright owner(s) are credited and that the original publication in this journal is cited, in accordance with accepted academic practice. No use, distribution or reproduction is permitted which does not comply with these terms. 